\title{
Are Smartphones Better in Guiding Physical Activity Among Sedentary Young Adults? A Randomised Controlled Trial
}

\author{
B. Tulasiram, B. Chandrasekaran \\ Centre for Sports Science, Medicine and Research, School of Allied Health Sciences, Manipal Academy of Higher \\ Education, Karnataka, India
}

\author{
CORRESPONDING AUTHOR: \\ Baskaran Chandrasekaran \\ Department of Exercise \\ and Sports Sciences \\ Manipal College of Health Professions \\ Manipal Academy of Higher Education \\ Madhav Nagar, Manipal \\ 576104 Karnataka, India \\ E-mail: baskaran.c@manipal.edu
}

DOI:

10.32098/mltj.01.2021.10

LEVEL OF EVIDENCE: 2B

\begin{abstract}
SUMMARY
Background. Physical activity promotion in young adults is found to be associated with improved psychosocial well-being and academic performance. Recently smartphones are found to be a potent means for promoting physical activity. So far no study has compared smartphones and traditional walking prescriptions in improving functional capacity and compliance in college adults.

Methods. Of 77 participants recruited, only 26 adult sedentary undergraduates with step count less than 7500 steps/ day were completed one of the two interventions: American College of Sports Medicine (ACSM) guided walking $(\mathrm{n}=14)$ and smartphone application (SMART) guided walking $(n=12)$. The pre and post-intervention oxygen uptake $\left(\mathrm{VO}_{2}\right)$, anaerobic threshold $\left(\mathrm{AT}\right.$ at $\left.\mathrm{VO}_{2}\right)$, heart rate $(\mathrm{HR})$ and ventilatory equivalents of carbondioxide $\left(\mathrm{V}_{\mathrm{E}} / \mathrm{VCO}_{2}\right)$ were measured by metabolimeter ( $\mathrm{K} 5$, Cosmed, Italy). After normalization, continous variables of $\mathrm{VO}_{2}, \mathrm{AT}$ at $\mathrm{VO}_{2}, \mathrm{~V}_{\mathrm{E}}$ ' $\mathrm{VCO}_{2}, \mathrm{HR}$ were analysed by two way analysis of variance (2 X 2 ANOVA) at a level of significance of 0.05 .

Results. Twenty six participants completed the study. Except treadmill distance and time, all exercise test variables including aerobic capacity $(3.20 \mathrm{ml} / \mathrm{kg} / \mathrm{min}, \mathrm{d}=0.492)$, anaerobic threshold $(-11.03, \mathrm{~d}=0.769)$, ventilatory equivalent of carbon dioxide $(2.32$ $\mathrm{ml} / \mathrm{kg} / \mathrm{min}, \mathrm{d}=0.801)$, heart rate $(-10.50 \mathrm{~b} / \mathrm{min}, \mathrm{d}=0.0792)$ improved significantly. Compliance was found to be $17.65 \%$ greater in the SMART than ACSM group. Conclusions. Physical activity promotion is efficient with SMART group than routinely administered traditional exercise prescription in improving the functional capacity of sedentary adults. Long-term compliance may be better with smartphone guided exercise prescription.
\end{abstract}

\section{KEY WORDS}

Physical activity; maximal aerobic capacity; smartphone; sedentary behaviour

\section{INTRODUCTION}

According to the Global Action Plan for Physical activity (2018-2030) proposed by World Health Organization, the prevalence of physical inactivity incidence is high in Low Middle-Income Countries (LMIC) including India (1). When compared to adults, the prevalence of physical inactivity is higher in adolescents with a drop in the activity level during the transition from school to college $(2,3)$. This low physical activity may be due to poor infrastructure, credit pressures and traditional seated academic curriculum in developing countries (2).
Physical activity (PA) implementation in schools and colleges has shown to improve quality of life and academic performance, and prevent cardio-metabolic risk in later life (2). Habitual PA is found to reduce frailty in muscle-tendon system, degeneration (4) and recently found to predict sarcopenia in older adults too (5). PA, in the form of walking, cycling and playing during breaks can be easily prescribed and integrated into school and college setups to reduce physical inactivity (6). Over the years, PA is prescribed using the metabolic calculations guided by American College of Sports Medicine (ACSM). These calculations are based on 
the maximal oxygen uptake $\left(\mathrm{VO}_{2}\right.$ max $)$ values measured by carrying out demanding tests in a well-established setup using open-circuit calorimetry (7). Exercise intensity ranging between $50 \%-85 \%$ of individual $\mathrm{VO}_{2}$ max value is prescribed to maintain or enhance cardiorespiratory fitness. This traditional method is followed for the past two decades but may fail in promotion of cardiorespiratory fitness due to poor compliance (8).

Recently, Smartphone applications ( $\mathrm{SmPh} A \mathrm{Ap})$ are found to influence human behaviour through social networking, self-motivation and goal-setting (6, 9). SmPh Apps such as Google Fit are designed to automatically track step counts and distance with an inbuilt magnetometer and global positioning system (GPS) respectively. Google Fit is a user-friendly application as it is simple, free of cost, with informal feedback and networking options (10). Few studies have been conducted to validate the Smartphone accelerometers in measuring step count $(7,8,11)$. However, Google Fit has not been used by researchers as an intervention to measure and influence PA in sedentary adults.

We conducted a pilot trial to investigate the effective way to prescribe PA based on compliance and change in functional capacity in college-going adults. The aim of the study was also to compare the effect of SmPh App (SMART) and traditional (ACSM) walking prescription on functional capacity on cardiorespiratory fitness of college-going adults.

\section{METHODS}

The study was conducted at the Department of Exercise and Sports Science of Manipal Academy of Higher Education after ethical approval from the Kasturba Medical College and Hospital Institutional Ethics Committee. The trial was also registered prospectively under the Clinical Trial Registry of India (CTRI/2018/01/011605). All participants were recruited after obtaining informed written consent. All the procedures were conducted according to the research ethics as described in the Declaration of Helsinki, and as required by the journal (12). The research study was completed in two phases. The study was planned and conducted, taking into consideration the examination schedule of all the students.

\section{Phase I}

\section{Participants}

Healthy adult volunteers of both the genders of age group 18-25 years, having android smartphones were recruited through advertisements across the university campus and Facebook. Group familiarization session was conducted to explain the study design and Google FIT application usage.
Students with any cardiovascular, pulmonary, and neuromuscular diseases affecting functional capacity and participants with recent surgeries, and musculoskeletal pain > $3 / 10$ on the visual analogue scale were excluded from the study. Besides, the presence of any diagnosed depression and history of taking anti-anxiety or anti-psychotic drugs were also included in the exclusion criteria.

\section{Measurement}

Average daily step count was measured using Smartphone-based (SmPh) application 'Google FIT'. We found a moderate to high validity of the step count in $\mathrm{SmPh}$ app ( $\mathrm{r}$ $=0.83$ ) when compared to a gold standard triaxial accelerometer (Actipal, PAL technologies, Glasgow, United Kingdom) on 12 male undergraduate volunteers in (an unpublished) pilot study conducted by us. The mean $\mathrm{SmPh}$-app based pedometer step count is found to have less error $(z$ $=-2.59 ; \mathrm{p}<0.01)$ compared to accelerometer steps $(\mathrm{cpm})$ on two different treadmill (calibrated) walk speeds (3 kmph and $5 \mathrm{kmph}$ ). During the monitoring period, male volunteers were requested to carry their smartphones in the right trouser pocket, while female students were given a pouch to strap to the right thigh. They were instructed to carry the mobile phone throughout the day except while bathing and sleeping. The step count (primary accelerometer data) was monitored over seven days, and everyday step count and the non-wear time was logged into an activity diary. The primary investigator collected the snapshots of the participant's weekly averaged step count with individuals coded on the WhatsApp group.

\section{Phase II}

\section{Participants}

Based on the data collected in the Phase I, young adults leading sedentary life [with characteristics of step-count less than 7500 steps from the average daily smartphone step count $(10,13)$, low aerobic capacity $\left(\mathrm{VO}_{2}\right)<35$ and 40 $\mathrm{ml} / \mathrm{kg} / \mathrm{min}$ for females and males respectively (14)] were included into the study. Measurement of $\mathrm{VO}_{2} \max$ quantification is explained in the measurement section below. The volunteers randomised to Exercise testing dates were rescheduled if female participants had menstrual periods.

\section{Maximal aerobic capacity $\left(\mathrm{VO}_{2}\right.$ max) measurement}

The maximal aerobic capacity $\left(\mathrm{VO}_{2} \max \right)$ was assessed by administering a modified Bruce treadmill protocol on all participants using indirect calorimeter (K5, Cosmed, Italy, 2014). The portable metabolimeter was calibrated for flow, 
reference gas and delay as per manufacturer standards. All participants were familiarised to treadmill $(\mathrm{h} / \mathrm{p}$ cosmos quasar, Italy) walking and the study protocol before administering the baseline measurement. Treadmill ergometer was calibrated for speed and grade as per earlier reliability study (15) prior the exercise testing of each participant. As per the modified Bruce protocol, students had to walk or run on the treadmill at incremental speeds and inclinations after warming up. Speed or inclination of the graded exercise test progressed after every 3 mins and the protocol was as follows: $2.7 \mathrm{~km} / \mathrm{hr}$ with $0 \%$ inclination, $2.7 \mathrm{~km} / \mathrm{h}$ at $5 \%, 2.7 \mathrm{~km} / \mathrm{h}$ at $10 \%, 4 \mathrm{~km} / \mathrm{hr}$ at $12 \%, 5.5 \mathrm{~km} / \mathrm{hr}$ at $14 \%$ and $6.8 \mathrm{~km} / \mathrm{hr}$ at $16 \%$. The criteria to terminate the test was: 1) plateau in $\mathrm{VO}_{2} ; 2$ ) Respiratory Exchange Ratio $(\mathrm{RER})>1.10 ; 3)$ Heart Rate $(\mathrm{HR})>95 \%$ HRmax (age-predicted maximal heart rate: $208-0.7 \times$ age (16)); and 4) Rate of Perceived Exertion (RPE) on Borg Scale more than 16. Participants were encouraged throughout the test to push themselves till the point of exhaustion. $\mathrm{VO}_{2}$ was collected breath by breath through telemetric portable metabolimeter (K5, Cosmed, Italy) and then transferred to the Omnia software. Peak heart rate (HR peak), maximal aerobic capacity $\left(\mathrm{VO}_{2}\right.$ peak), treadmill test duration $(\mathrm{min})$ and stage of Modified Bruce Protocol at which exhaustion or $\mathrm{VO}_{2}$ peak achieved were noted from the software after data reduction. After completion of four weeks of intervention, the aerobic capacity $\left(\mathrm{VO}_{2}\right)$ using the metabolimeter was again measured using a modified Bruce treadmill exercise testing protocol. Peak heart rate (HR peak), maximal aerobic capacity $\left(\mathrm{VO}_{2}\right.$ peak), treadmill test duration ( $\mathrm{min}$ ) and stage of Modified Bruce Protocol at which exhaustion or $\mathrm{VO}_{2}$ peak achieved were analysed for intervention effects.

\section{The sample size for intervention phase}

To get a minimum detectable difference of at least $1 \mathrm{ml} / \mathrm{kg} /$ min in $\mathrm{VO}_{2}$ at self-selected speeds (17), at least 24 participants were needed to attain a power of $80 \%$ and level of significance $95 \%$ allowing dropout of $20 \%$ using the formula $\left(Z_{\alpha / 2}+Z_{\beta}\right)^{2} * 2 * \sigma^{2} / \mathrm{d}^{2}$ where $\sigma$ is $0.47 \mathrm{ml} / \mathrm{kg} / \mathrm{min}$ and $\mathrm{d}$ is 0.6. After analysing step count and maximal aerobic capacity $\left(\mathrm{VO}_{2} \max \right), 29$ sedentary college students were included in the study.

\section{Randomization}

Participants were randomly allocated into two intervention groups: ACSM and SMART, using computer-generated randomization. Sealed envelopes disclosing the intervention group details were opened after the participants signed the informed consent.

\section{Intervention}

This was a four-weeks interventional study, comparing the functional capacity of the participants who received traditional (ACSM) and smartphone-based (SMART) exercise prescription. A familiarisation session was given regarding ACSM based exercise intensity and step count progression for four weeks. The student volunteers were randomly allocated to ACSM based exercise prescription (ACSM) group and Smartphone-based exercise prescription (SMART) group. Both the groups were provided with the diary log with tabulated exercise prescription and attendance sheet for validation of the compliance.

\section{SMART group}

For the Google FIT app-based group, we considered 7,500 step/day as the baseline for less active category (13). To improve or maintain cardiovascular health, recent evidence finds 10000 steps/day to be a valid criterion measure and globally recommended practise(18). Participants from the SMART group were requested to walk 8500 steps/day in the first week of intervention. Later 1000 steps/day were added every week till completion of four weeks of intervention. Thus, in the final week of intervention, students were requested to walk 11,500 steps/day thus reaching the global recommendations (18). The $\mathrm{SmPh}$ app is designed to automatically track the step count per day, and the primary investigator monitored weekly average step count. All participants were given weekly feedback regarding the progression of step count through a short message service (SMS) and WhatsApp. Weekly progression of step count was monitored by the primary investigator through WhatsApp group with participant coded rather than names.

\section{ACSM group}

In the case of ACSM group, the walking regimen was prescribed based on their $\mathrm{VO}_{2}$ peak achieved by the individuals during pre-maximal exercise testing. We applied the ACSM running equation to estimate the walking distance and time for all students.

ACSM running equation: $V_{2}(\mathrm{ml} . \mathrm{kg}-1 . \mathrm{min}-1)=(0.2 \times S)+$ $(0.9 \times S \times G)+3.5$ ml.kg-1.min-1.

(Where, $S=$ Speed and $G=$ Grade). When walking on ground level (flat), $\mathrm{G}=0$. Thus the final equation is: $\mathrm{VO}_{2}$ (ml. $\mathrm{kg}-1$. min-1 $)=(0.2 \times S)+3.5$ ml.kg-1.min-1.

Week wise speed calculation:

- for first week, $\mathrm{S} 1=50 \%$ to $60 \%$ of $\mathrm{VO}_{2}\left(\mathrm{ml} \cdot \mathrm{kg}^{-1} \cdot \mathrm{min}^{-1}\right)$ $3.5\left(\mathrm{ml}^{\mathrm{kg}} \mathrm{kg}^{-1} \cdot \mathrm{min}^{-1}\right) / 0.2$.

- for second week, $\mathrm{S} 2=60 \%$ to $70 \%$ of $\mathrm{VO}_{2}\left(\mathrm{ml} . \mathrm{kg}^{-1}\right.$. $\left.\min ^{-1}\right)-3.5\left(\mathrm{ml} \cdot \mathrm{kg}^{-1} \cdot \mathrm{min}^{-1}\right) / 0.2$.

- for third week, $\mathrm{S} 3=70 \%$ to $80 \%$ of $\mathrm{VO}_{2}\left(\mathrm{ml} \cdot \mathrm{kg}^{-1} \cdot \mathrm{min}^{-1}\right)$ $-3.5\left(\mathrm{ml} \mathrm{kg}^{-1} \cdot \mathrm{min}^{-1}\right) / 0.2$. 
- for fourth week, $\mathrm{S} 4=80 \%$ to $90 \%$ of $\mathrm{VO}_{2}\left(\mathrm{ml} \cdot \mathrm{kg}^{-1} \cdot \mathrm{min}^{-1}\right)$ $-3.5\left(\mathrm{ml}^{\mathrm{kg}} \mathrm{kg}^{-1} \cdot \mathrm{min}^{-1}\right) / 0.2$.

Target distance to be walked (meters) $=$ Target speed $(\mathrm{m} /$ $\mathrm{min}) /$ minute. Target distance in 30 minutes = Target distance in one-minute x 30 minutes. All the participants were prescribed walking distance based on individual basis. All participants were requested to walk the prescribed distance every day for 30 minutes for four weeks consecutively, with a gradual increment in the prescribed distance they had to cover in 30 minutes. This walking prescription was within the range of $50 \%$ to $90 \%$ of individual $\mathrm{VO}_{2} \max$ At least $80 \%$ of attendance in the activity log was included for analysis.

\section{STATISTICAL ANALYSIS}

Continuous measures such as step count, aerobic capacity $\left(\mathrm{VO}_{2}\right)$, ventilatory equivalent of carbondioxide $\left(\mathrm{V}_{\mathrm{E}} \mathrm{CO}_{2}\right)$, anaerobic threshold $\left(\mathrm{AT}\right.$ at $\mathrm{VO}_{2}$ ), treadmill distance and time were summarized as mean and standard deviations using descriptive statistics. The distribution of baseline variables of subjects who were lost to follow-up were assumed due to random factors. Per protocol analysis was followed for missing data. Raw data was tested for normality of distribution (Shapiro Wilk test) and homogeneity of variance (Levene's test). As the data was normally distributed, two-way repeated measures ANOVA was administered to compare intervention groups (SMART \& ACSM) with timing of the interventions (PRE and POST). Mixed-effects models were used to study changes in $\mathrm{VO}_{2}, \mathrm{~V}_{\mathrm{E}} \mathrm{CO}_{2}$, AT, TD and TT related to group (SMART; ACSM), time, and group $\times$ time interaction, adjusting for baseline demographic variables. If significant effects were observed, post-hoc comparisons were performed as appropriate, with Bonferroni corrections for multiple comparisons. The treatment effect size will be estimated using Cohen's 'd'. Cohen's $d=$ $\left(M_{2}-M_{1}\right) / S D_{\text {pooled }}$ where $S D_{\text {pooled }}=\sqrt{ }\left(\left(S D_{1}^{2}+S D_{2}^{2}\right) / 2\right)$ where $\mathrm{M}_{2}, \mathrm{M}_{1}$ and $\mathrm{SD}_{1}, \mathrm{SD}_{2}$ are mean differences and standard deviations among the intervention groups, respectively. The preference among SMART and ACSM group towards interventions was assessed using the Mann Whitney $U$ test. A p value of less than 0.05 was considered statistically significant. Data were analyzed using the statistical package, SPSS 22.0 (IBM, Chicago).

\section{RESULTS}

Out of 132 volunteers, only $29(21.97 \%)$ sedentary adults [7 males and 22 females] were found eligible for the study based on the baseline measures: step count $(<7.500$ steps/ day) and $\mathrm{VO}_{2}$ values $\left(\mathrm{VO}_{2}\right.$ peak $<35 \mathrm{ml} / \mathrm{kg} / \mathrm{min}$ in females and $<40 \mathrm{ml} / \mathrm{kg} / \mathrm{min}$ males), and entered into the randomization. The overall flow of the participants is shown in the CONSORT diagram (figure 1).

Out of 29 students, 26 (SMART group $\mathrm{n}=12$; ACSM group $\mathrm{n}=14$ ) adults completed the four weeks intervention program, and we used their data for statistical analysis. In our study, $35.06 \%$ of females and $9.09 \%$ of males were sedentary (figure 2).

Out of 17 participants in the ACSM group, 14 completed $(82.35 \%)$ whereas all 12 participants in the SMART group $(100 \%)$ completed the intervention. Preference towards SMART intervention was statistically significant than ACSM physical activity intervention $(\mathrm{Z}=3.086 ; \mathrm{p}=0.006)$.

The baseline characteristics of the 26 participants completed; both interventions are demonstrated in table $\mathbf{I}$.

\section{Effect of type and timing of interventions}

The interaction (group $\mathrm{x}$ time) effects showed a statistically significant difference among $\mathrm{VO}_{2}, \mathrm{~V}_{\mathrm{E}} \mathrm{CO}_{2}, \mathrm{AT}$ at $\mathrm{VO}_{2}$ and HR $(\mathrm{p}<0.05)$ whereas no difference is noted in treadmill time and distance (table III). Only main effects showed statistically significant difference in TT $[\mathrm{f}=6.09(1.26)]$ and TD $[f=4.04$ (1.26) $]$ among interventions (table III). There

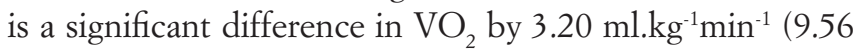
$\%), \mathrm{V}_{\mathrm{E}} \mathrm{CO}_{2}$ slope by $11.03, \mathrm{AT}^{2}$ at $\mathrm{VO}_{2}$ by $2.32 \mathrm{ml} / \mathrm{kg} / \mathrm{min}$ and HR by 11 beats/min $(\mathrm{p}<0.05)$ whereas TD and TT did not show any significant difference among interventions (table II). We also found a moderate effect size $(0.4-0.8)$ in SMART than ACSM group among the exercise testing variables as seen in table II. Figure 3 depicts the changes among the significant exercise testing variables.

\section{DISCUSSION}

We found smartphone-based PA intervention improves functional capacity $\left(\mathrm{VO}_{2} \max \& \mathrm{~V}_{\mathrm{E}} \mathrm{CO}_{2}\right)$ and fatigue levels (treadmill time and anaerobic threshold) than traditional metabolic calculation driven physical activity prescription.

\section{Effect of technology-based intervention on exercise testing variables}

In our study, after four weeks of walking intervention, we found an average of $9 \%$ increase in $\mathrm{VO}_{2}$ in smartphone-based PA prescription group than ACSM based PA prescription. We hypothesise that the determinants such as goal setting and motivation might have improved the compliance towards the prescribed step count and reflected improved $\mathrm{VO}_{2}, \mathrm{~V}_{\mathrm{E}} \mathrm{CO}_{2}$ and fatigue variables (AT, TD) (19). 


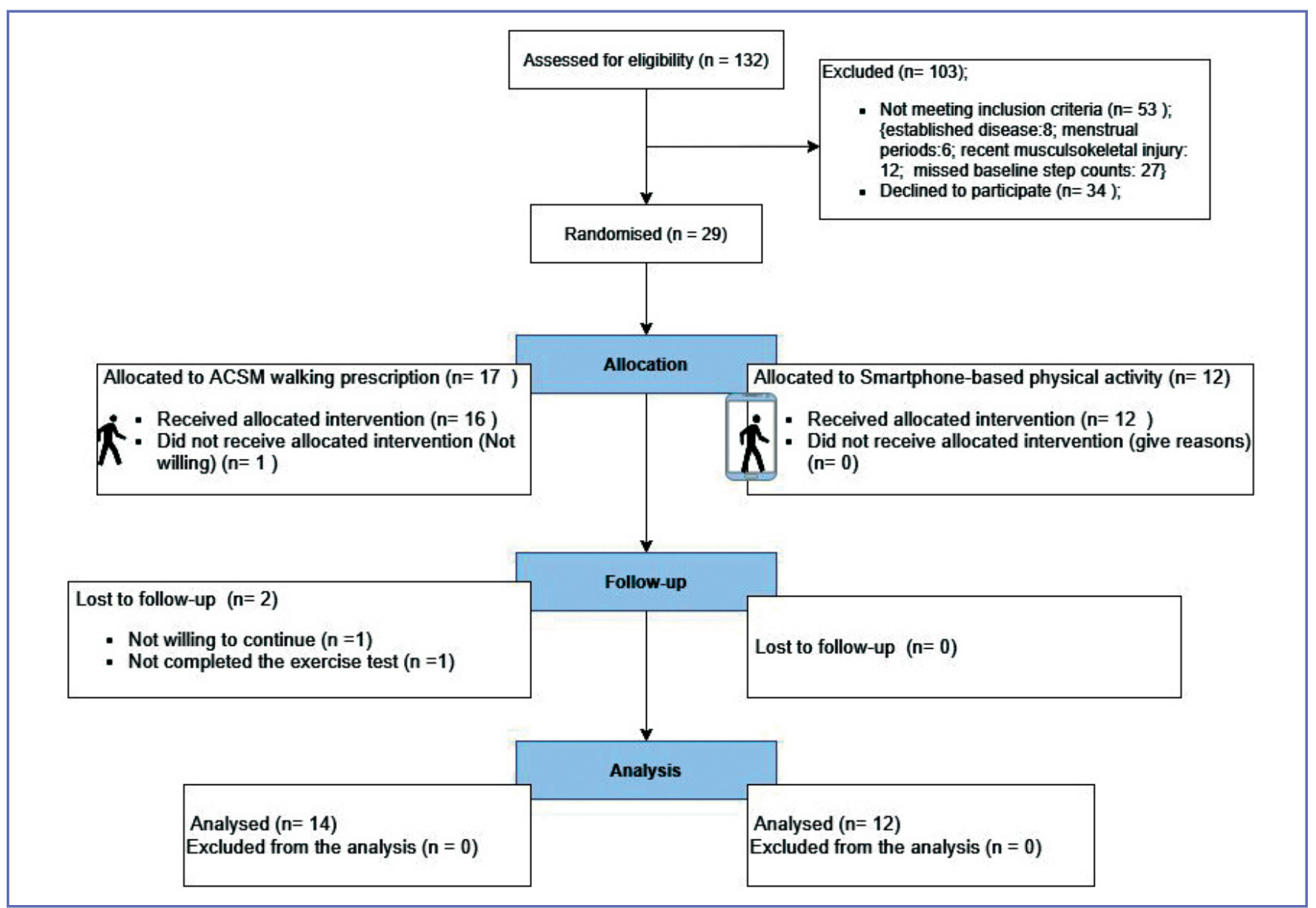

Figure 1. CONSORT diagram showing the recruitment of participants.

We propose smartphone based physical activity promotion may be an appropriate behavioral intervention satisfying the theory of planned behavior and reasoned action (20). We agree to the earlier studies that has found PA associated with muscular, ligament structural adaptations (21), cardiovascular dynamics thereby improving $\mathrm{VO}_{2}, \mathrm{~V}_{\mathrm{E}} \mathrm{CO}_{2}$ slope and anaerobic threshold. We did not find any significant difference in the treadmill time and distance. We hypothesised that our both interventions focused on the walking, thus might not have caused any effect on the walking distance or time. If we had a control group, we might have mitigated this insignificant finding.

\section{Compliance towards PA intervention}

Most of our study participants preferred smartphone-based PA intervention than traditional PA intervention. Thus, we agree with (22) that PA administered using smartphone applications may improve long-term adherence. Hence, to gain the maximum benefits like reduction in risk of cardiometabolic disorders and improvement in psychosocial well-being and quality of life, it is vital to be compliant with the optimal amount of PA regimen (23).

\section{Prevalence of sedentary behavior}

We found that 1 in 2 females and 1 in 5 males of college adults are sedentary in our study population. Our study results comply with the ICMR-INDIAB study (24) that $54.4 \%$ were inactive ( $58.3 \%$ females). Probable reasons for inactivity in females may be due to perceived incompetence and lack of family support towards sporting behaviour in female college students (25).

\section{Strengths of our study}

1) To our knowledge, this is the first Indian study to address sedentary behaviour of college students and to demonstrate 


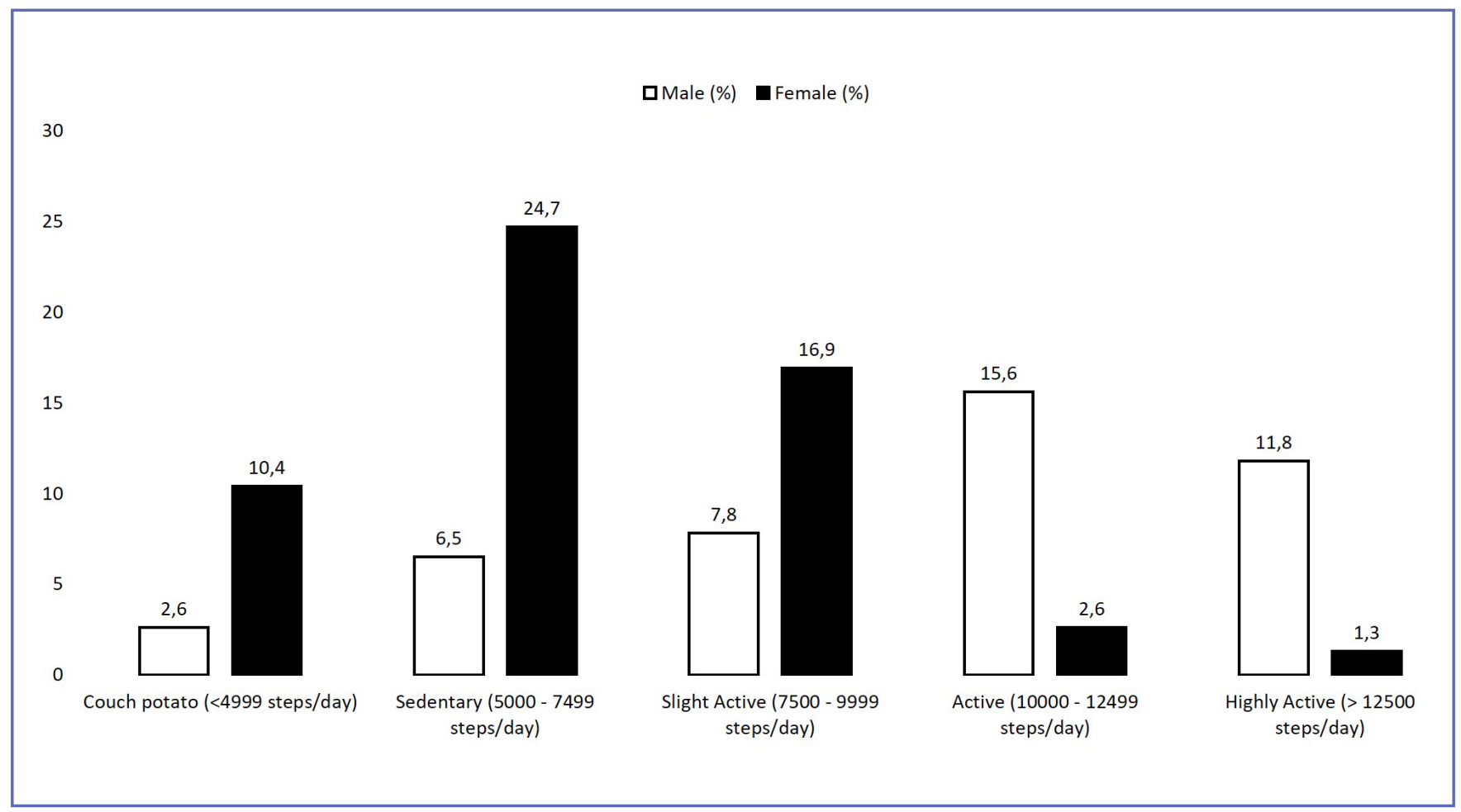

Figure 2. Prevalence of sedentary behaviour (<7500 steps/day) in participants.

Table I. Baseline characteristics of all participants.

\begin{tabular}{|c|c|c|c|}
\hline \multirow{2}{*}{ Variables } & SMART Group $(n=12)$ & ACSM Group $(n=14)$ & \multirow{2}{*}{$\begin{array}{l}\text { Difference } \\
(P<0.05)\end{array}$} \\
\hline & Mean \pm SD & Mean \pm SD & \\
\hline Age (years) & $22 \pm 2$ & $21 \pm 4$ & 0.314 \\
\hline Gender (n) & Male (3); Female (9) & Male (4); Female (10) & - \\
\hline Body Mass Index & $23.3 \pm 1.82$ & $22.7 \pm 2.34$ & 0.265 \\
\hline Diet (Kcal) & $2130 \pm 236$ & $1984 \pm 474$ & 0.368 \\
\hline $\begin{array}{c}\text { Aerobic capacity } \\
\left(\mathrm{VO}_{2} \text { in } \mathrm{ml} / \mathrm{kg} / \mathrm{min}\right)\end{array}$ & $28.92 \pm 5.25$ & $28.46 \pm 3.28$ & 0.735 \\
\hline Treadmill distance $(\mathrm{m})$ & $679.50 \pm 50.62$ & $750.07 \pm 89.65$ & 0.241 \\
\hline
\end{tabular}

SMART: Smartphone application-based break reminders; ACSM: American College of Sports Medicine prescribed physical activity group.

the efficacy, feasibility of introducing a social cognitive model-based intervention to improve PA level. 2) Upcoming smartphone application-based (technology-assisted) physical activity prescription is compared to the traditional gold standard physical activity prescription method. 3) Dropouts very minimal with the Smartphone guided walking prescription, which is distinct finding supporting behavioral theoretical framework.

\section{The weakness of our study}

Our study lacks control group that reduced the isolated effect of smartphone-based PA intervention in sedentary young adult community. We recommend future studies to compare the smartphone based behavioral promotion with control wait list group to isolate the effects of the intervention Our study was administered in a single multifaceted university to generalize the results to the young adults 
Table II. Post hoc between-group comparisons and effect size for event related potentials (ERPs) that showed significant interaction between intervention and electrodes.

\begin{tabular}{|c|c|c|c|c|c|c|}
\hline \multirow{2}{*}{ Electrodes } & \multirow[t]{2}{*}{ Task } & \multicolumn{5}{|c|}{ Mean \pm SD } \\
\hline & & Pre & Post & Pre vs post & SMART vs ACSM & Cohen's d \\
\hline \multirow{2}{*}{$\begin{array}{l}\text { Aerobic capacity }\left(\mathrm{VO}_{2}\right. \\
\text { in } \mathrm{ml} / \mathrm{kg} / \mathrm{min})\end{array}$} & ACSM & $28.64 \pm 3.28$ & $29.96 \pm 4.76$ & $1.50 \pm 3.72 *$ & $3.20 \pm 4.02 * *$ & 0.492 \\
\hline & SMART & $28.17 \pm 4.60$ & $33.58 \pm 3.92$ & $5.42 \pm 2.50 * *$ & & \\
\hline \multirow{2}{*}{$\mathrm{V}_{\mathrm{E}} \mathrm{CO}_{2}($ slope $)$} & ACSM & $33.94 \pm 2.52$ & $33.34 \pm 2.24$ & $-0.60 \pm 2.14^{*}$ & $-11.03 \pm 3.98^{* * *}$ & 0.769 \\
\hline & SMART & $34.16 \pm 2.66$ & $29.43 \pm 2.11$ & $-4.73 \pm 2.50 * *$ & & \\
\hline \multirow{2}{*}{$\begin{array}{l}\text { Anerobic threshold } \\
\left(\mathrm{VO}_{2} \text { at } \mathrm{AT}\right)\end{array}$} & ACSM & $13.48 \pm 1.69$ & $14.42 \pm 2.49$ & $0.94 \pm 1.76$ & $2.32 \pm 2.26^{*}$ & 0.801 \\
\hline & SMART & $14.99 \pm 1.75$ & $17.71 \pm 1.70$ & $2.72 \pm 0.93$ & & \\
\hline \multirow{2}{*}{$\begin{array}{l}\text { Treadmill } \\
\text { distance (meters) }\end{array}$} & ACSM & $751.07 \pm 89.65$ & $759.04 \pm 91.00$ & $7.96 \pm 36.24$ & $17.00 \pm 6.33$ & 0.451 \\
\hline & SMART & $679.50 \pm 50.62$ & $750.46 \pm 23.60$ & $70.96 \pm 57.32$ & & \\
\hline \multirow{2}{*}{$\begin{array}{l}\text { Treadmill } \\
\text { time (minutes) }\end{array}$} & ACSM & $13.34 \pm 0.93$ & $13.54 \pm 0.91$ & $0.21 \pm 0.49$ & $1.56 \pm 0.77$ & 0.457 \\
\hline & SMART & $12.63 \pm 0.51$ & $13.22 \pm 0.43$ & $0.59 \pm 0.63$ & & \\
\hline \multirow{2}{*}{$\begin{array}{l}\text { Heart Rate } \\
\text { peak } \\
\text { (beats/min) }\end{array}$} & $\mathrm{ACSM}$ & $174.14 \pm 5.27$ & $171.93 \pm 3.81$ & $-2.21 \pm 5.34$ & $-10.50 \pm 5.97 *$ & 0.792 \\
\hline & SMART & $173.83 \pm 4.20$ & $165.25 \pm 4.22$ & $-8.58 \pm 3.87$ & & \\
\hline
\end{tabular}

$\mathrm{V}_{\mathrm{E}} \mathrm{CO}_{2}$ - Ventilatory equivalent of Carbon dioxide; Heart Rate ${ }_{\text {peak }}-$ Maximal Heart rate occurred during the stress test; SMART - Smartphone based physical activity promotion; ACSM - American College of Sports Medicine; '*' represents $\mathrm{p}<0.050$; ' $* *$ ' represents $\mathrm{p}<0.010$.

Table III. Results of 2*2 (intervention*time) analysis of variance analyses for exercise testing variables.

\begin{tabular}{|c|c|c|c|c|c|c|}
\hline \multirow[t]{2}{*}{ Variable } & \multicolumn{2}{|c|}{ Main effect of intervention } & \multicolumn{2}{|c|}{ Main effect of time } & \multicolumn{2}{|c|}{$\begin{array}{l}\text { Interaction effects } \\
\text { (intervention*time) }\end{array}$} \\
\hline & F Value (df) & P value & F Value (df) & P value & F Value (df) & $P$ value \\
\hline Aerobic capacity $\left(\mathrm{VO}_{2}\right)$ & $2.84(1.26)$ & $0.022 *$ & $6.49(1.26)$ & $0.014 *$ & $1.721(1.26)$ & $0.010 * *$ \\
\hline $\mathrm{V}_{\mathrm{E}} \mathrm{CO}_{2}($ slope $)$ & $7.66(1.26)$ & $0.008^{* * *}$ & $16.00(1.26)$ & $0.000 * *$ & $9.611(1.26)$ & $0.003 * *$ \\
\hline Anerobic threshold $\left(\mathrm{VO}_{2}\right.$ at $\left.\mathrm{AT}\right)$ & $19.54(1.26)$ & $0.000 * *$ & $11.35(1.26)$ & $0.001 * *$ & $2.70(1.26)$ & $0.015^{*}$ \\
\hline Treadmill distance (meters) & $4.04(1.26)$ & $0.049 *$ & $3.92(1.26)$ & 0.053 & $2.50(1.26)$ & 0.121 \\
\hline Treadmill time (minutes) & $6.09(1.26)$ & $0.017 *$ & $3.60(1.26)$ & 0.062 & $0.858(1.26)$ & 0.359 \\
\hline Heart Rate peak (beats/min) & $8.05(1.26)$ & $0.007 * *$ & $19.22(1.26)$ & $0.000 * *$ & $6.89(1.26)$ & $0.013 *$ \\
\hline
\end{tabular}

$\overline{\mathrm{V}_{\mathrm{E}} \mathrm{CO}_{2} \text { - Ventilatory equivalent of Carbon dioxide; Heart Rate }}{ }_{\text {peak }}-$ Maximal Heart rate occurred during the stress test; SMART - Smartphone based physical activity promotion; ACSM - American College of Sports Medicine; F - Fischer's value of ANOVA; 'a' represents $\mathrm{p}<0.050$; ' $\mathrm{b}$ ' represents $\mathrm{p}<0.010$.

around the globe. However, we tried to limit this weakness through appropriate randomization. Further, we attained the sample size to illustrate the needed change in the aerobic capacity after the intervention. We also used westernized metabolic equations to establish the speed/ distance of the walking prescription, which may not be appropriate for the Indian population.

\section{Limitations}

Despite strengths, few limitations worth addressing are: 1) though familiarized, the sedentary adults recruited in this study were not very well acquainted to walk on the tread- mill. Thus the $\mathrm{VO}_{2}$ values obtained from the graded maximal exercise test was their peak and not maximal. 2) Furthermore, we had to use the ACSM running equation to estimate walking speed in our study, which may not be appropriate for the Indian population. Future Indian trials should establish the validity for estimating the appropriate speed/ distance of walking through ACSM equations. 3) Smartphone-based position and activity monitoring are improving with time but still is not foolproof, wholly accurate and reliable. Future randomized controlled trials should target a larger sample and administer customized applications at varied positions for accurate measurement and influence of long-term adherence to PA program in the community. 

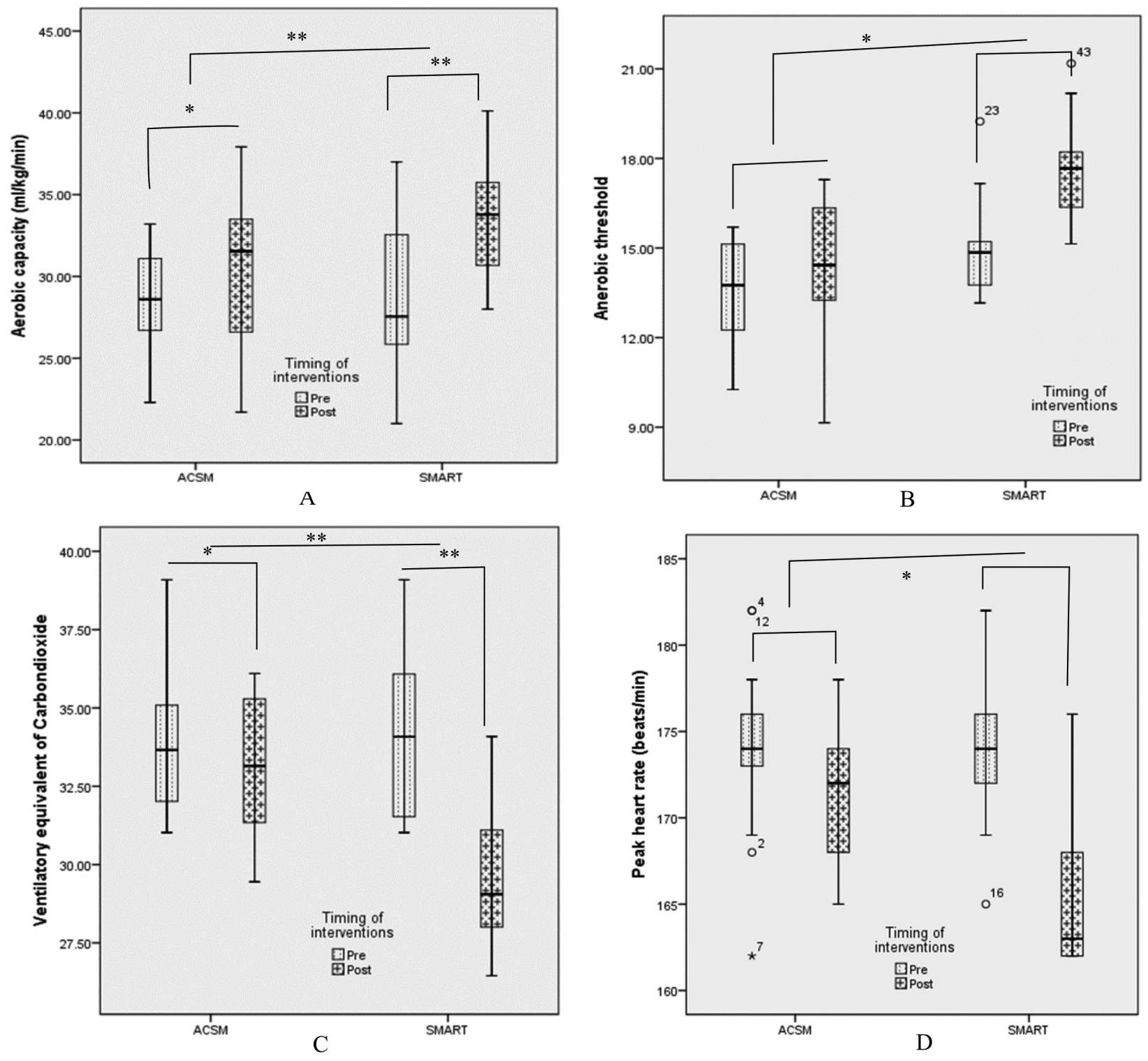

Figure 3. Boxplots demonstrating the significant differences in maximal exercise test variables among interventions: A. Aerobic capacity ( $\mathrm{ml} / \mathrm{kg} / \mathrm{min})$. B. Anerobic Threshold ( $\mathrm{ml} / \mathrm{kg} / \mathrm{min})$. C. Ventilatory equivalent of carbondioxide slope and D. Peak Heart Rate (beat/min).

\section{CONCLUSIONS}

We conclude that PA prescription using smartphone application is more effective in improving functional capacity when compared to the traditional way of exercise prescription and PA promotion. Therefore, PA can be introduced, prescribed, promoted and improved effectively with better compliance for long-term functional and health benefits using smartphone applications as a medium.

\section{ETHICS}

All procedures performed in studies involving human participants were in accordance with the ethical standards of the institutional and/or national research committee and with the 1964 Helsinki declaration and its later amendments or comparable ethical standards. 


\section{CONTRIBUTORS}

$\mathrm{BC}$ conceived and designed the research, TR carried out the participant recruitment, data collection and follow-up of clients. Both TR and BC interpreted the findings and drafted and proofread the manuscript.

\section{ACKNOWLEDGEMENTS}

The authors wish to thank Dr Fiddy Davis PhD, Head of the Department, Department of Exercise and Sports Sciences, Manipal Academy of Higher Education, Manipal, Karnataka, India for his continuous support and motivation for the research and manuscript. The authors wish to thank Ms Purva Gandhi, MPT, Private Practitioner for her intellectual inputs and language editing in the manuscript.

\section{CONFLICT OF INTERESTS}

The authors declare that they have no conflict of interests.

\section{REFERENCES}

1. Nugent R, Bertram MY, Jan S, et al. Investing in non-communicable disease prevention and management to advance the Sustainable Development Goals. Lancet 2018;391(10134):2029-35.

2. Watson A, Timperio A, Brown H, Best K, Hesketh KD. Effect of classroom-based physical activity interventions on academic and physical activity outcomes: a systematic review and meta-analysis. Int J Behav Nutr Phys Act 2017;14(1):114.

3. Bort-Roig J, Martin M, Puig-Ribera A, et al. Uptake and factors that influence the use of 'sit less, move more' occupational intervention strategies in Spanish office employees. Int J Behav Nutr Phys Act 2014;11(1):152-61.

4. Bezerra MA, da Silva Nery C, de Castro Silveira PV, et al. Previous physical exercise slows down the complications from experimental diabetes in the calcaneal tendon. MLTJ 2016;6(1):97-103.

5. Agnes T, Vishal K, Girish N. Regression model for the prediction of risk of sarcopenia among older adults. MLTJ 2019;9(3):425-32.

6. Lewis BA, Napolitano MA, Buman MP, Williams DM, Nigg CR. Future directions in physical activity intervention research: expanding our focus to sedentary behaviors, technology, and dissemination. J Behav Med 2017;40(1):112-26.

7. Toledo MJ, Hekler E, Hollingshead K, Epstein D, Buman M. Validation of a Smartphone App for the Assessment of Sedentary and Active Behaviors. JMIR Mhealth Uhealth 2017;5(8):e119.

8. Silsupadol P, Teja K, Lugade V. Reliability and validity of a smartphone-based assessment of gait parameters across walking speed and smartphone locations: Body, bag, belt, hand, and pocket. Gait Posture 2017;58:516-22.

9. Bort-Roig J, Gilson ND, Puig-Ribera A, Contreras RS, Trost SG. Measuring and influencing physical activity with smartphone technology: a systematic review. Sports Med 2014;44(5):671-86.

10. Melo X, Santa-Clara H, Santos DA, et al. Linking cardiorespiratory fitness classification criteria to early subclinical atherosclerosis in children. Appl Physiol Nutr Metab 2015;40(4):386-92.

11. Hekler EB, Buman MP, Grieco L, et al. Validation of Physical Activity Tracking via Android Smartphones Compared to ActiGraph Accelerometer: Laboratory-Based and Free-Living Validation Studies. JMIR Mhealth Uhealth 2015;3(2):e36.

12. Padulo J, Oliva F, Frizziero A, Maffulli N. Basic principles and recommendations in clinical and field science research: 2018 update. MLTJ 2018;8(3):305-7.

13. Tudor-Locke C, Craig CL, Thyfault JP, Spence JC. A step-defined sedentary lifestyle index: <5000 steps/day. Appl Physiol Nutr Metab 2013;38(2):100-14.

14. Scholl J, Bots ML, Peters SA. Contribution of cardiorespiratory fitness, relative to traditional cardiovascular disease risk factors, to common carotid intima-media thickness. J Intern Med 2015;277(4):439-46.

15. Padulo J, Chamari K, Ardigò LP. Walking and running on treadmill: the standard criteria for kinematics studies. MLTJ 2014;4(2):159-62.

16. Tanaka H, Monahan KD, Seals DR. Age-predicted maximal heart rate revisited. J Am Coll Cardiol 2001;37(1):153-6.

17. Darter BJ, Rodriguez KM, Wilken JM. Test-retest reliability and minimum detectable change using the K4b2: oxygen consumption, gait efficiency, and heart rate for healthy adults during submaximal walking. Res Q Exerc Sport 2013;84(2):223-31.

18. Al-Kuwari MG, Al-Mohannadi AS, Sayegh S. Effectiveness of "Step into Health" program in Qatar: a pedometer-based longitudinal study. J Sports Med Phys Fitness 2017;57(11):1513-8.

19. Bond DS, Thomas JG, Raynor HA, et al. B-MOBILE - A Smartphone-Based Intervention to Reduce Sedentary Time in Overweight/Obese Individuals: A Within-Subjects Experimental Trial. PLoS One 2014;9(6): e100821.

20. Zhao J, Freeman B, Li M. Can Mobile Phone Apps Influence People's Health Behavior Change? An Evidence Review. J Med Internet Res 2016;18(11):e287.

21. Coombes B, Tucker K, Hug F, et al. Relationships between cardiovascular disease risk factors and Achilles tendon structural and mechanical properties in people with Type 2 Diabetes. MLTJ 2019;9(3):395-404.

22. Stuckey MI, Carter SW, Knight E. The role of smartphones in encouraging physical activity in adults. Int J Gen Med 2017;10:293-303.

23. Norton LH, Norton KI, Lewis NR. Adherence, Compliance, and Health Risk Factor Changes following Short-Term Physical Activity Interventions. Biomed Res Int 2015;2015:929782.

24. Anjana RM, Pradeepa R, Das AK, et al. Physical activity and inactivity patterns in India - results from the ICMR-INDIAB study (Phase-1) [ICMR-INDIAB-5]. Int J Behav Nutr Phys Act 2014;11(1):26.

25. Deliens T, Deforche B, De Bourdeaudhuij I, Clarys P. Determinants of physical activity and sedentary behaviour in university students: a qualitative study using focus group discussions. BMC Public Health 2015;15:201. 